\title{
Glycogen metabolism and Cori cycle in human hair follicles
}

Katarzyna Figlak1, Ralf Paus ${ }^{2}$, Mike Philpott'

2Dermatology Res Ctr, Inst. Of Inflammation \& Repair, University of Manchester, Manchester, UK

\section{BACKGROUND}

The hair follicle (HF) is a dynamic mini-organ that continuously undergoes phases of growth (anagen), apoptosis-driven regression (catagen), and relative quiescence (telogen).

Hair follicles have one of the highest rates of cell division in the human body and metabolise $90 \%$ of glucose to lactate. Catabolism of glucose to lactate is considered energetically inefficient, but in actively proliferating cells catabolism of glucose to lactate generates a high flux of metabolites that can be tapped for biosynthesis of proteins, nucleic acids and lipids and thus contribute to biomass and growth control.

Cori cycle is a metabolic process mainly occurring in the liver, in which excess lactate is converted into glucose through gluconeogenesis to prevent lactic acidosis. Glucose is then used for glycogen replenishment.

Glycogen is osmotically neutral glucose polymer found mostly in the liver and skeletal muscle, but also in hair follicles.

The aim of this study is to determine the presence and localisation of glycogen and glycogen metabolism enzymes in human hair follicles and to dissect the possibility of the Cori cycle operating in the hair follicle.

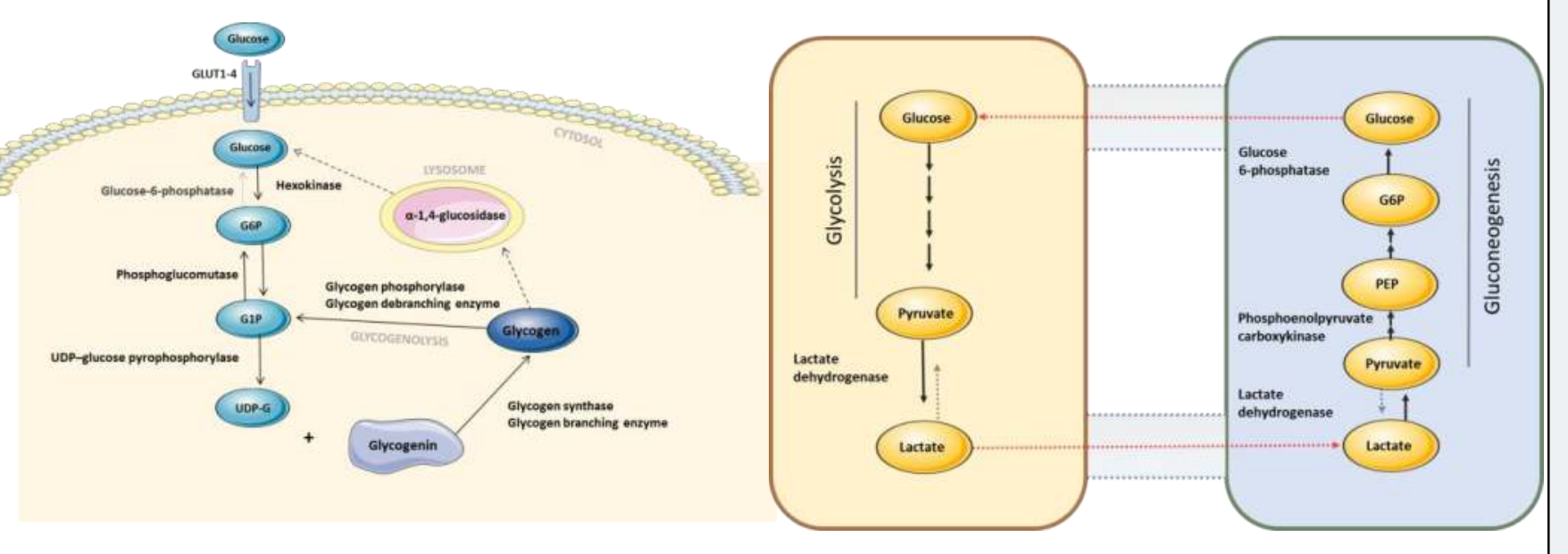

\section{RESULTS}

1 Glycogen is present in the ORS of human hair follicles

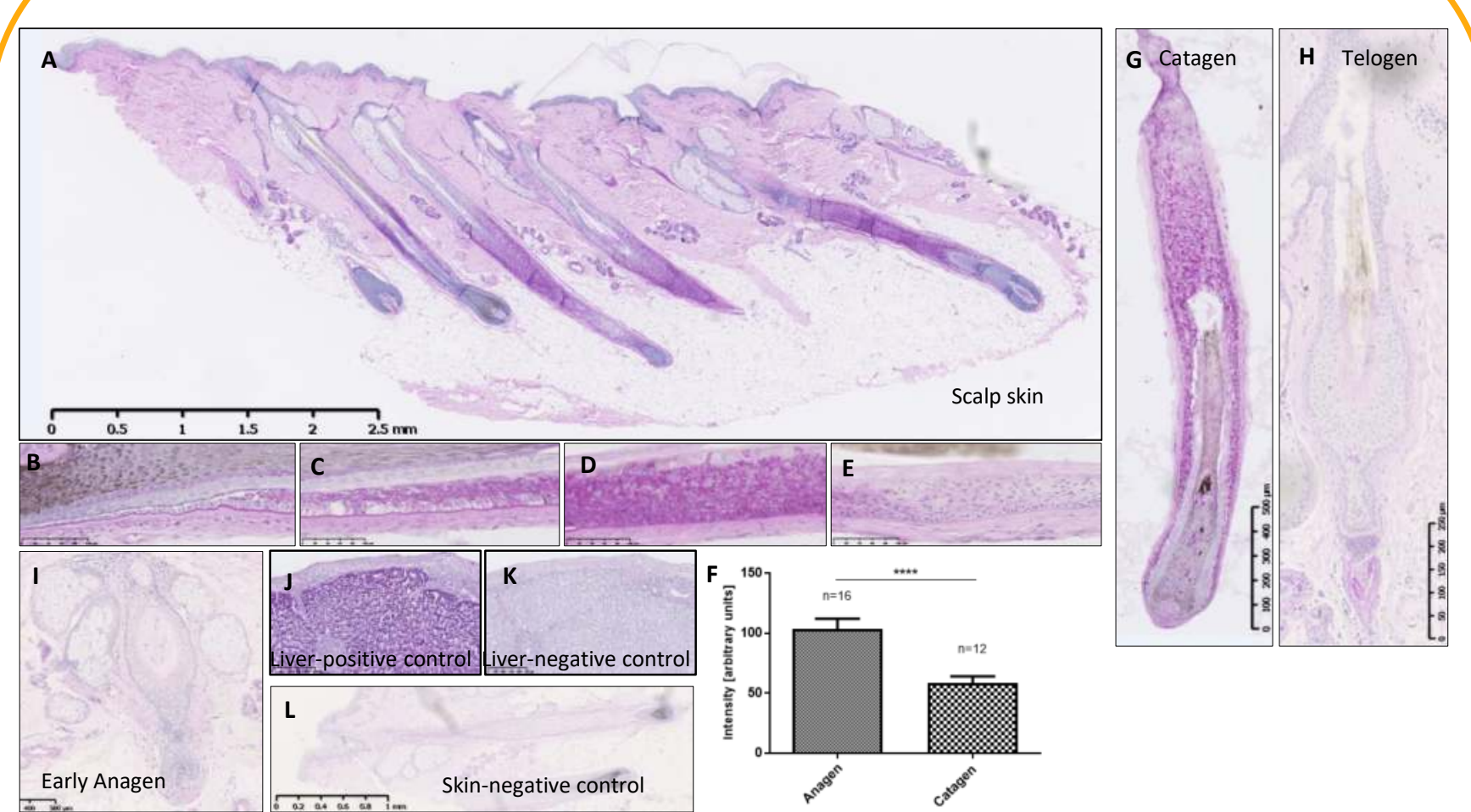

Figure 1 Glycogen presence: Periodic Acid Schiff staining (magenta glycogen staining) in (A)-human scalp section; B-Eisolated anagen hair follicles from the bulb (B), suprabulbar region (C, D) and bulge region (E). (F)-staining intensity quantification in anagen and catagen hair follicles (**** P<0.0001). (G)-PAS staining in catagen hair follicle (cultured in
vitro for 5 days). Scalp skin sections with telogen $(H)$ and early anagen (I) hair follicles, Liver sections stained for vitro for 5 days). Scalp skin sections with telogen (H) and early anagen (I) hair follicles. Liver sections stained for
glycogen as a positive control (J); (K)-negative control (digestion with $\alpha$-amylase from porcine pancreas) in the liver and glycogen as a positive control (J); (K)-negative control (digestion with $\alpha$-amylase from porcine pancreas) in the liver and

2
(2)
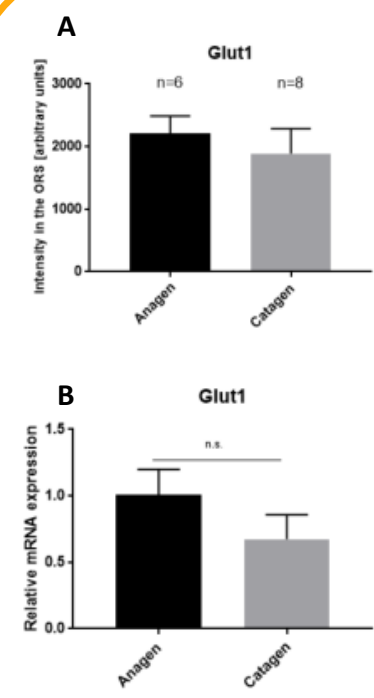

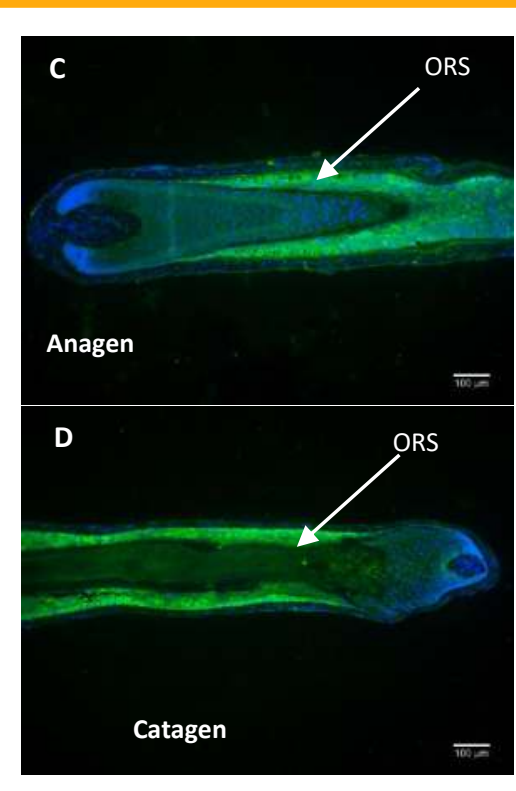

Glucose transporter 1 is expressed in the ORS

2 Expression of glucose transporter 1 (Glut1) (A)-immunofluorescence staining quantification in the RRS in anagen and catagen hair follicles, (B)-relative mRNA $(n=2)$, normalised to B2M, n.s. $p>0.05$. (nmunofluorescence performed in isolated anagen (C) and catagen (D, cultured in vitro for six days) hair follicles using

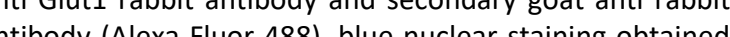
with DAPI. Scale bars dente 100 n.

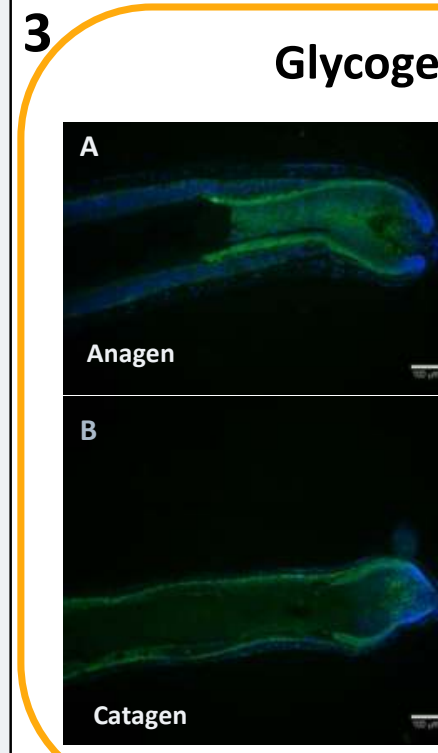

Glycogen synthase 1 is expressed in the IRS and basal ORS

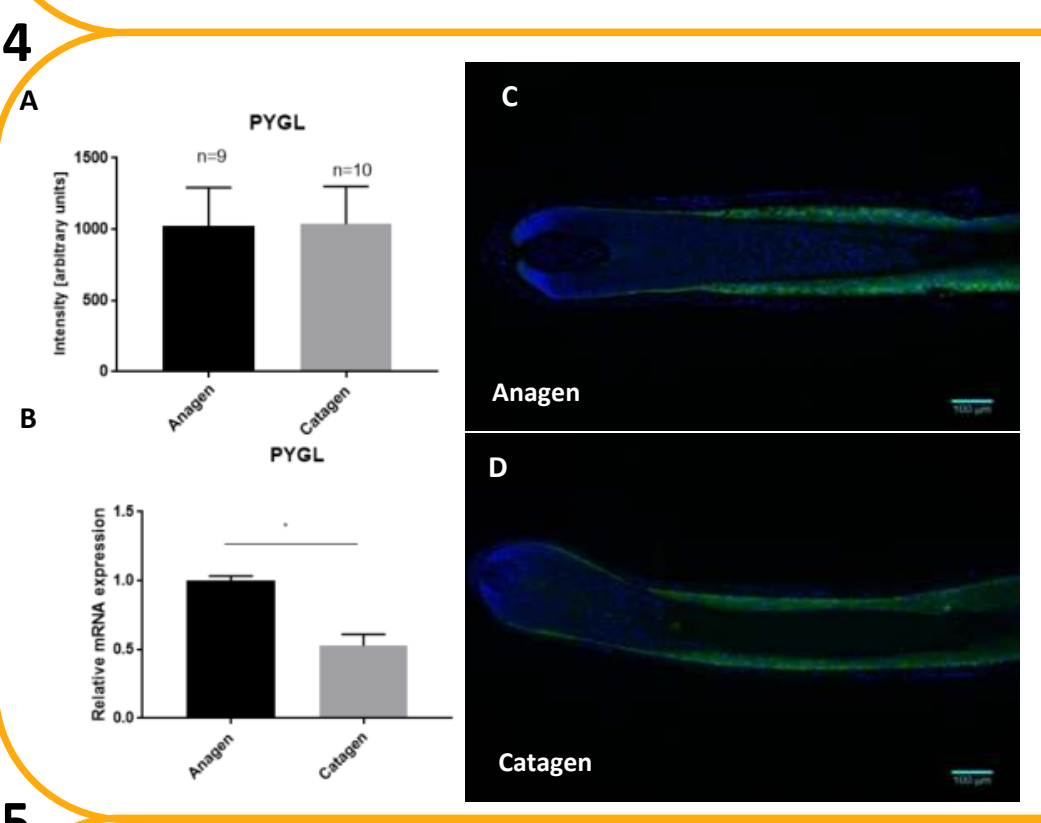

Glycogen phosphorylase is expressed in the ORS Figure 4 Expression of glycogen phosphorylase (PYGL)

(A)-staining quantification in the ORS in anagen and catagen hair follicles, (B)-relative mRNA expression of PYGL in anagen and catagen hair
follicles $(n=2)$, normalised to $B 2 \mathrm{M},{ }^{*} \quad p<0.05$. Immunofluorescence performed in isolated anagen (C) and catagen (D, cultured in vitro for
six days) hair follicles using anti Glut1 rabbit antibody and secondary goat anti rabbit antibody (Alexa Fluor 488), blue nuclear staining obtained with DAPI. Scale bars denote $100 \mu \mathrm{m}$.

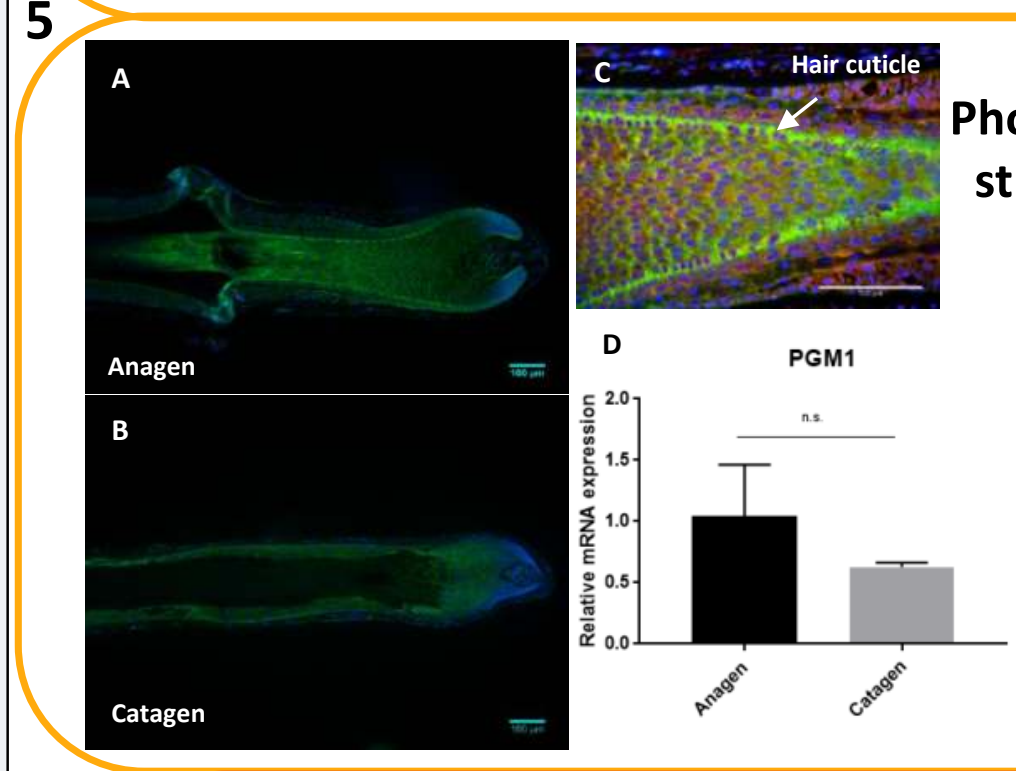

Phosphoglucomutase 1 presents the strongest expression in the cuticle Figure
(PGM1)

Immunofluorescence performed in isolated anagen (A) and catagen (B, cultured in vitro for six days) hair follicles using anti PGM1 rabbit antibody and
secondary goat anti rabbit antibody (Alexa Fluor secondary goat anti rabbit antibody (Alexa Fluor
488), blue nuclear staining obtained with DAPI. (C)zoomed image of suprabulbar region stained with PGM1 in anagen and catagen hair follicles $(n=2)$ normalised to B2M, n.s. p p 0.05 . 6

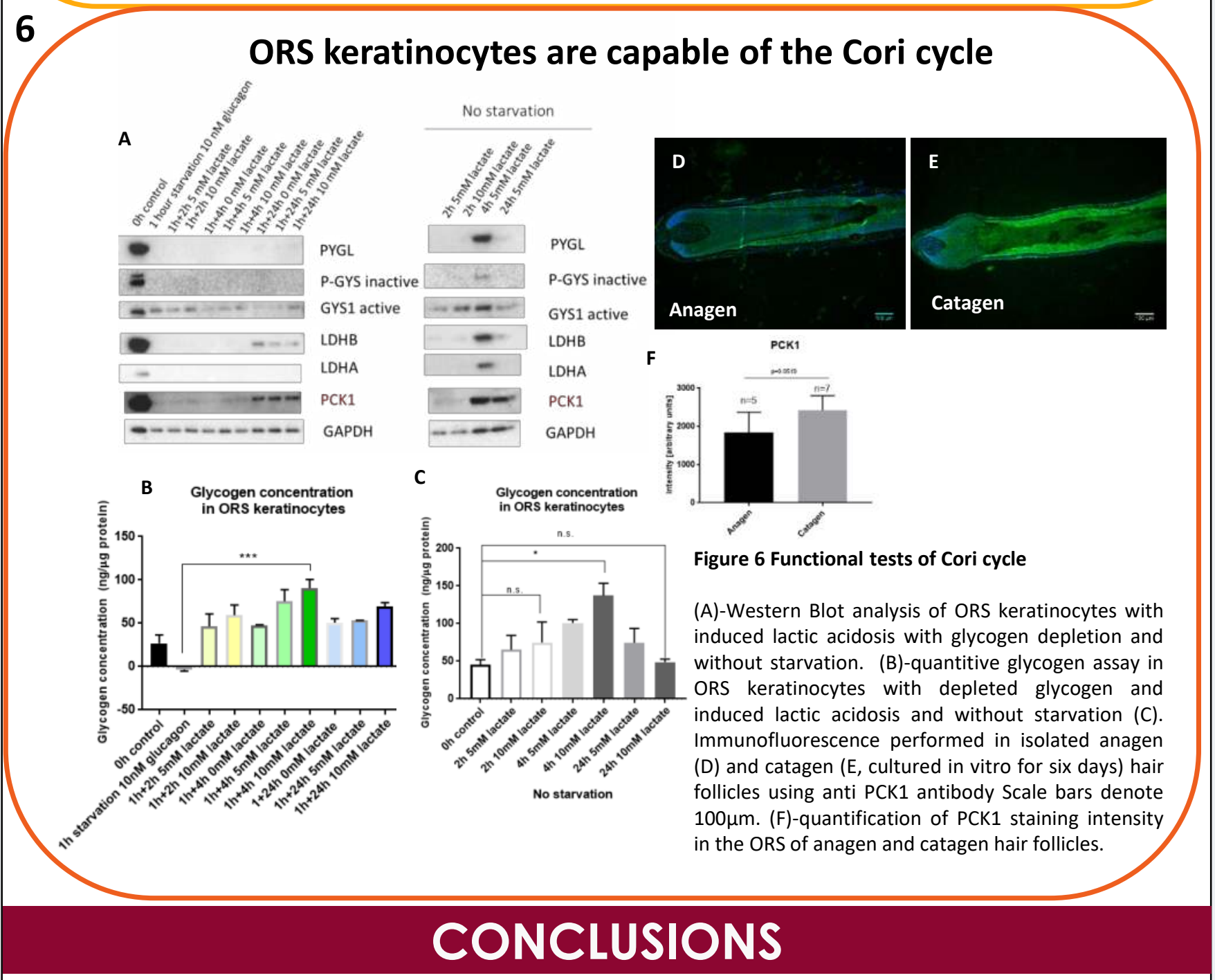

Hair follicles contain high amounts of glycogen

Glycogen level changes with hair follicle growth phases

Glycogen metabolism enzymes present distinct expression patterns in hair follicles Glycogen-source of energy, precursors for keratinisation?

Hair follicles express crucial gluconeogenetic enzyme-PCK1

Do ORS keratinocytes produce glycogen to prevent lactic acidosis?

Is hair follicle the liver of the skin? 